\title{
Emotionally Intelligent Organizations
}

\author{
Adrienn Cseresznyés \\ Széchenyi István University
}

\begin{abstract}
The life of the organization is determined by the manners of its members and the quality of its relationships. The organization does not have emotions, but employees being part of the organization express emotions during their life in the workplace. Emotional intelligence that influences human relationships can be interpreted at an organizational level. The emotionally intelligent company acknowledges the emotional nature of interactions with stakeholders and understands that adapting emotional decision making to their daily routine makes a competitive advantage over those companies who might not attach importance to emotional manifestations. This conceptual paper aims to clarify the attributes of an emotionally intelligent company by implementing the model of Trait Emotional Intelligence Questionnaire (TEIQue) by K.V. Petrides. The possible outcome of the research is to assess an organization's emotional intelligence level through employees and to determine different organizational profiles with EI.
\end{abstract}

Keywords: organizational development, EI, learning organization, organizational culture, leadership 